\section{International Scientific Journal Theoretical \& Applied Science}

p-ISSN: $2308-4944$ (print)

e-ISSN: 2409-0085 (online)

Year: 2016

Issue: 3

Volume: 35

Published: 30.03 .2016

http://T-Science.org

SECTION 2. Applied mathematics. Mathematical modeling.
Alexandr Shevtsov

candidate of technical sciences, member of PILA (USA),

member of European Academy of Natural History (UK), member of American Academy of Applied Sciences,

Department of «Mathematics»,

Taraz state University named after M.Kh. Dulati,

Kazakhstan

$\underline{\text { Shev_AlexXXXX@mail.ru }}$

Sara Ashimovna Suleymenova teacher of mathematics

Taraz College of engineering and business,

\title{
SOME ERROR COMPUTER ALGEBRA SYSTEMS WHEN SOLVING SYSTEMS OF INEQUALITIES
}

Abstract: Discusses several cases of errors in the answers, when solving systems of inequalities in the system of computer algebra.

Key words: Computer algebra system, error, inequalities.

Language: English

Citation: Shevtsov AN, Suleymenova SA (2016) SOME ERROR COMPUTER ALGEBRA SYSTEMS WHEN SOLVING SYSTEMS OF INEQUALITIES. ISJ Theoretical \& Applied Science, 03 (35): 189-192.

Soi: http://s-o-i.org/1.1/TAS-03-35-33 Doi: crossef http://dx.doi.org/10.15863/TAS.2016.03.35.33

When solving systems of inequalities with application of computer algebra systems there are some errors that lead to incorrect results in the response. Consider the basic command for solving inequalities.

The command solve () is used to solve inequalities and systems of inequalities in real numbers exactly the same as for solving equations and systems of equations [1-5]. The answer is expressed either as a set of inequalities, either through function RealRange () and Open (). The first specifies a closed interval of real numbers, and the second is used to specify that the boundary point is not included in the constructed solution. To specify the solution as a set, should be set as a set of either inequality or unknown, regarding which the decision is searched. If this is not done, then the answer will be obtained using these functions in determining the actual intervals.

Example solution of inequalities:

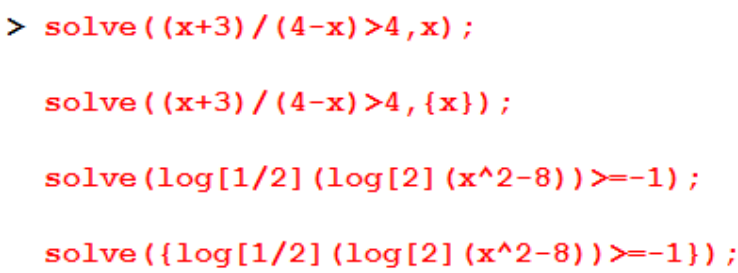

In this example, solved two inequalities, for each of which the solution is constructed as a set and in the form of real intervals. As observed there is no problem. Now consider several systems of equations [6-10]. 


\begin{tabular}{|c|c|c|c|c|c|c|}
\hline Impact Factor: & $\begin{array}{l}\text { ISRA (India) } \\
\text { ISI (Dubai, UAF } \\
\text { GIF (Australia) } \\
\text { JIF }\end{array}$ & $\begin{array}{l}=1.344 \\
=0.829 \\
=0.564 \\
=1.500\end{array}$ & $\begin{array}{l}\text { SIS (USA) } \\
\text { PИНЦ (Russia) } \\
\text { ESJI (KZ) } \\
\text { SJIF (Morocco) }\end{array}$ & $\begin{array}{l}=0.912 \\
=0.179 \\
=1.042 \\
=2.031\end{array}$ & $\begin{array}{l}\text { ICV (Poland) } \\
\text { PIF (India) } \\
\text { IBI (India) }\end{array}$ & $\begin{array}{l}=6.630 \\
=1.940 \\
=4.260\end{array}$ \\
\hline
\end{tabular}

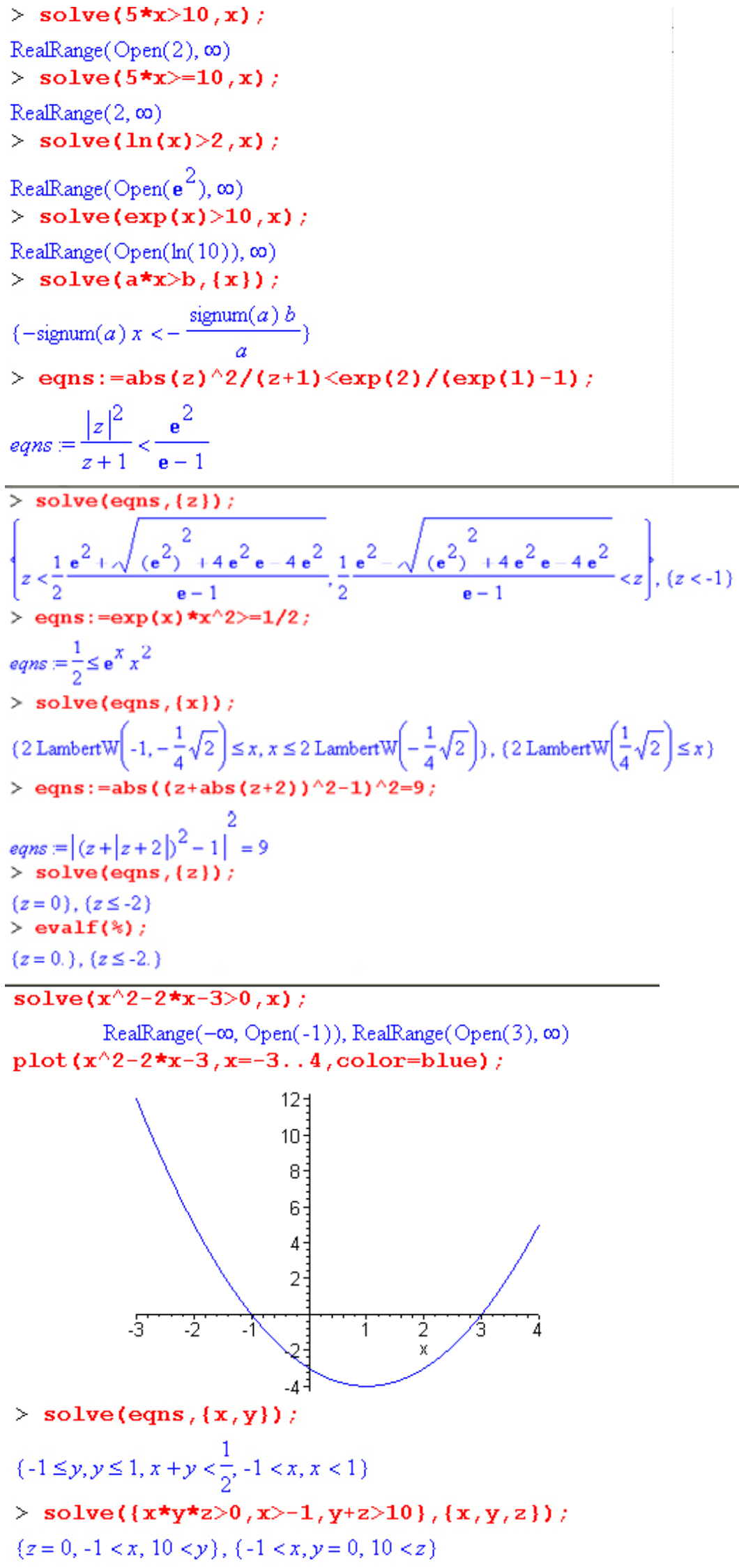

As observed there is no problem. Define the system of inequalities: 


\begin{tabular}{l|lrl|l|ll} 
& ISRA (India) & $=\mathbf{1 . 3 4 4}$ & SIS (USA) & $=\mathbf{0 . 9 1 2}$ & ICV (Poland) & $=\mathbf{6 . 6 3 0}$ \\
Impact Factor: & ISI (Dubai, UAE) $=\mathbf{0 . 8 2 9}$ & PUHL (Russia) $=\mathbf{0 . 1 7 9}$ & PIF (India) & $=\mathbf{1 . 9 4 0}$ \\
& GIF (Australia) & $\mathbf{0 . 5 6 4}$ & ESJI (KZ) & $=\mathbf{1 . 0 4 2}$ & IBI (India) & $=\mathbf{4 . 2 6 0}$ \\
& JIF & $\mathbf{1 . 5 0 0}$ & SJIF (Morocco) & $=\mathbf{2 . 0 3 1}$ & & \\
\hline
\end{tabular}

restart;

d1 : $=2 * x-5<=0$;

d2: $=x * x-5 * x+6<=0$;

d3: $=x * x-5 * x+6>=0$;

d4 : $=2 * x-5>=0$;

$\mathrm{r} 1:=\operatorname{evalf}($ solve $(\{\mathrm{d} 1, \mathrm{~d} 2\}, \mathrm{x}))$;

r2:=evalf (solve $(\{d 3, d 4\}, x))$;

Solve the system of inequalities:

$$
\begin{gathered}
d 1:=2 x-5 \leq 0 \\
d 2:=x^{2}-5 x+6 \leq 0 \\
d 3:=0 \leq x^{2}-5 x+6 \\
d 4:=0 \leq 2 x-5 \\
r 1:=\{2 . \leq x, x \leq 2.500000000\} \\
r 2:=\{3 . \leq x\}
\end{gathered}
$$

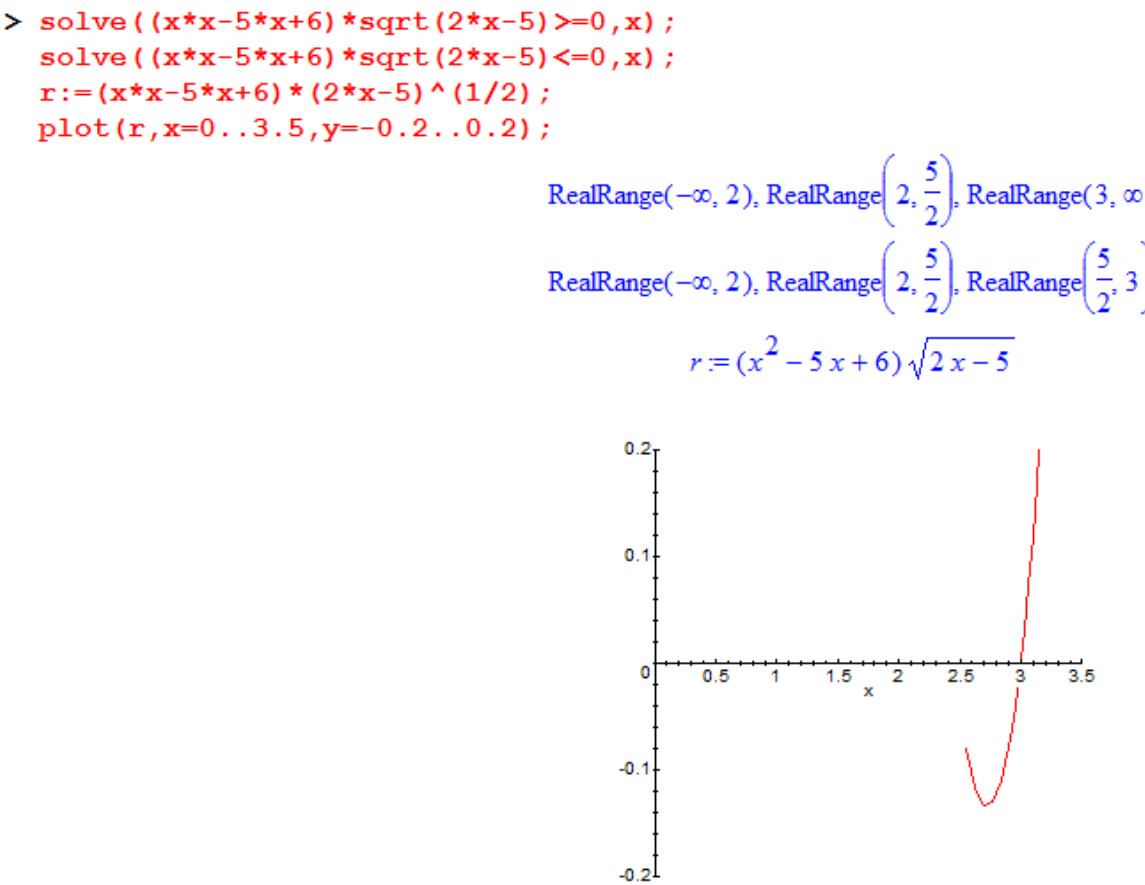

Observed for the first system of inequalities is the appearance of two gaps identifying where in reality function can not exist.

$$
\begin{aligned}
& \text { RealRange }(-\infty, 2) \text {, RealRange }\left(2, \frac{5}{2}\right), \\
& \operatorname{RealRange}(-\infty, 2), \operatorname{RealRange}\left(2, \frac{5}{2}\right),
\end{aligned}
$$

For the second system of inequalities observed a similar situation. 


\begin{tabular}{l|lrl|l|ll} 
& ISRA (India) & $=\mathbf{1 . 3 4 4}$ & SIS (USA) & $=\mathbf{0 . 9 1 2}$ & ICV (Poland) & $=\mathbf{6 . 6 3 0}$ \\
Impact Factor: & ISI (Dubai, UAE) $=\mathbf{0 . 8 2 9}$ & PUHL (Russia) $=\mathbf{0 . 1 7 9}$ & PIF (India) & $=\mathbf{1 . 9 4 0}$ \\
& GIF (Australia) & $\mathbf{0 . 5 6 4}$ & ESJI (KZ) & $=\mathbf{1 . 0 4 2}$ & IBI (India) & $=\mathbf{4 . 2 6 0}$ \\
& JIF & $\mathbf{1 . 5 0 0}$ & SJIF (Morocco) & $=\mathbf{2 . 0 3 1}$ & & \\
\hline
\end{tabular}

\section{References:}

1. (2016) Reshenie uravneniy, neravenstv i ikh sistem. Available: http://www.0zd.ru/programmirovanie kompyut ery_i/reshenie_uravnenij_neravenstv_i_ix.html (Accessed: 10.03.2016).

2. (2016) Prosteyshie preobrazovaniya i vychisleniya. Available: http://physics.herzen.spb.ru/library/03/02/maple tut/page3.html (Accessed: 10.03.2016).

3. (2016) Maple. Available: http://detc.1s.urfu.ru/Assets/aMATH0011/mp5.h tm (Accessed: 10.03.2016).

4. (2016) Neravenstva v Maple 9. Available: http://skorost5.ucoz.ru/metodrazrabotki/Xrustal ev/Maple/6konspekt uroka.doc (Accessed: 10.03.2016).

5. (2016) Programmnaya razrabotka biblioteki protsedur v srede Maple - kak faktor razvitiya umeniy programmirovaniya. Available: http://www.rankpedagogy.ru/inspuds-1693.html (Accessed: 10.03.2016).

6. (2016) Reshenie neravenstv i sistem neravenstv. Available: http://life-prog.ru/2_23014_reshenieneravenstv-i-sistem-neravenstv.html (Accessed: 10.03.2016).
7. Savotchenko SE, Kuz'micheva TG (2001) Metody resheniya matematicheskikh zadach $\mathrm{v}$ Maple. 2001.

8. (2016) Metodicheskoe posobie po Maple Available:

http://vtorokursnik.ru/index.php?first=7\&secon $\mathrm{d}=116 \&$ third=18531 (Accessed: 10.03.2016).

9. Kuz'micheva TG (2016) Metody resheniya matematicheskikh zadach v Maple. Available: http://gigabaza.ru/doc/73363-p2.html (Accessed: 10.03.2016).

10. (2016) Maple. Available: http://edu.dvgups.ru/METDOC/ENF/SAPR/IN FORMATIKA/ZAOCH/LABORATORNYE\% 20\%20RABOTY\%20PO\%20MAPLE\%20V\%2 OUSTANOVOChN.PDF (Accessed: 10.03.2016).

11. (2016) Maple. Available: http://window.edu.ru/catalog/pdf2txt/089/24089 16599?p_page $=3$ (Accessed: 10.03.2016).

12. (2016) Maple. Available: http://www.studfiles.ru/preview/2460803/page: 3/ (Accessed: 10.03.2016).

13. (2016) Reshenie neravenstv. Available: http://maple.plusby.com/Glava-8/Index29.htm (Accessed: 10.03.2016). 